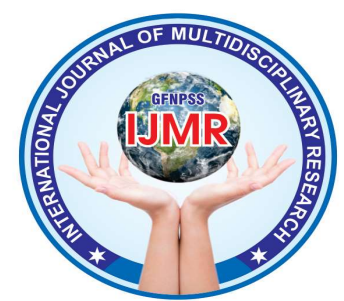

\title{
PREVALENCE \& CLINICAL SPECTRUM OF VITAMIN B12 DEFICIENCY
}

\section{ROHIT AVASTHI ${ }^{1}$, GIRIRAJ PRASAD SONI ${ }^{2}$}

${ }^{1} \mathrm{Ph} . \mathrm{D}$. Scholar, Mahatma Gandhi Nursing College, Sitapura, Jaipur

${ }^{2}$ Dean \& Principal Mahatma Gandhi Nursing College, Sitapura, Jaipur

Corresponding Email: avasthi.rohit@yahoo.co.in

\begin{abstract}
:
Vitamin $\mathrm{B}_{12}$ deficiency was report before 100 years ago, but still, we did not reach the final conclusion about correct diagnosis and right treatment. Various divert symptoms of vitamin B12 may be differ from neurologic to psychiatric symptoms. Many people suffer from vitamin B12 deficiency present with classic megaloblastic anaemia.
\end{abstract}

Keyword: Prevalence, Clinical spectrum, vitamin b12 deficiency

\begin{tabular}{ccc}
\hline Received & Accepted & Available online \\
\hline $29 / 07 / 2021$ & $03 / 08 / 2021$ & $05 / 08 / 2021$ \\
\hline
\end{tabular}

\section{INTRODUCTION:}

Deficiency of Vitamin B12 occurs mostly by macrocytic anaemia. The role of $\mathrm{B}_{12}$ deficiency in hyperhomocysteinemia (Increased homocysteine in the body) and advancement of atherosclerosis is currently explored. Vitamin $\mathrm{B}_{12}$ deficiency confirm by serum vitamin $B_{12}$ value; however, about 50 percent of patients with subclinical disease have normal $\mathrm{B}_{12}$ levels. Other techniques of screening for vitamin $\mathrm{B}_{12}$ deficiency is investigation of serum methylmalonic acid and homocysteine levels, which are increased early in vitamin $\mathrm{B}_{12}$ deficiency. Schilling test used for the detection of pernicious anaemia.Various research show that administration of vitamin B12 to oral route is an effective and safe treatment of vitamin $\mathrm{B}_{12}$ deficiency.

When intrinsic factor is not present to aid in the absorption of vitamin $\mathrm{B}_{12}$ or in other diseases that affect the usual absorption sites in the terminal ileum, oral therapy remains effective.

\section{CLINICAL MANIFESTATIONS:}

It indicated that vitamin $\mathrm{B}_{12}$ deficiency is also relate with hematologic, neurological, and psychiatric symptoms. Vitamin $B_{12}$ deficiency is a common cause of megaloblastic anaemia, in advanced cases, pancytopenia is the cause of vitamin B12 deficiency. Pathological condition resulting from vitamin $B_{12}$ deficiency such as paresthesias, peripheral neuropathy, and 


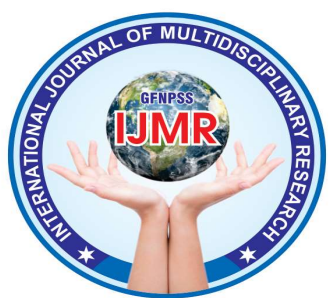

demyelination of the corticospinal tract and dorsal columns.

Various psychiatric disorders, such as impaired memory, irritation, depression, dementia and, sometimes psychosis also develop vitamin $\mathrm{B}_{12}$ deficiency.

\section{NORMAL ABSORPTION OF VITAMIN B:}

Only two enzymatic reactions are known to be dependent on vitamin $\mathrm{B}_{12}$ in human body. When the primary process begin methylmalonic acid is converted to succinyl-Co A using vitamin $\mathrm{B}_{12}$ as a cofactor. Vitamin $B_{12}$ deficiency, therefore lead to increased levels of serum methylmalonic acid. When secondary process begin, homocysteine is converted to methionine by using vitamin $\mathrm{B}_{12}$ and folic acid as cofactors. Because of this reaction a deficiency of vitamin $\mathrm{B}_{12}$ or folic acid may lead to increased homocysteine levels. Absorption cycle of vitamin $B_{12}$ provide help to illuminate the potential causes vitamin $\mathrm{B}_{12}$ deficiency.

Breakdown of vitamin $B_{12}$ that is bound to food due to the process of acidic environment of the stomach. Intrinsic factor, which is released by parietal cells in the stomach, binds to vitamin $\mathrm{B}_{12}$ in the duodenum and absorption of vitamin $\mathrm{B}$ 12 in the ileum

This vitamin $\mathrm{B}_{12}$-intrinsic factor complex subsequently aids in the absorption of vitamin
$\mathrm{B}_{12}$ at distal end of small intestine.

In addition to this method of absorption, various research show that existence of an alternate system that is independent of intrinsic factor or even an intact terminal ileum. Approximately 1 percent of a large oral dose of vitamin $\mathrm{B}_{12}$ is absorbed by this second mechanism. Once absorbed, vitamin $\mathrm{B}_{12}$ binds to transcobalamin II and is transported throughout the body. Development of any types of interruption of these steps places a person at risk of developing vitamin B12deficiency.

\section{HOW TO DIAGNOSIS OF DEFICIENCY} OF VITAMIN B 12:

$>$ Complete blood count

$>$ Vitamin B12 level

$>$ Schilling test.

$>$ Antibodies test.

$>$ Methylmalonic acid test

\section{CAUSES OF VITAMIN B 12 DEFICIENCY} STATES:

\section{$>$ Due to Deficient intake:}

Breastfeeding babies of mothers may develop vitamin B12 deficiency by age 4 to 6 months because in these babies, liver stores are limited and because of more growth depends on the high requirements.

Due to Inadequate absorption: This is common cause of vitamin B12 deficiency. 


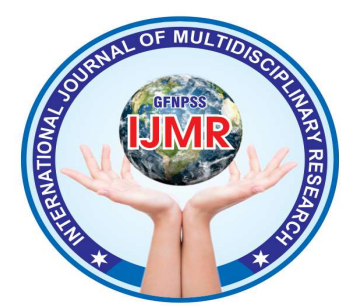

inadequate absorption due to decreased acid secretion.

> Due to Use of certain drugs: Due to certain drug which are responsible for vit. B12 deficiency such as Antacids, Metformin, Nitrous oxide

$>$ Due to Decreased utilization: Enzyme deficiencies, Liver disorders, Transport protein abnormality

\section{PREVENTION AND TREATMENT OF B12 DEFICIENCY:TREATMENT}

Cyanocobalamin can be administered by Intramuscular route or oral supplement of $\mathrm{B}_{12}$ therapy. Administered injection three times per week for two weeks in patients without neurologic deficits. In case of neurological deficit, injections should be given every other day for up to three weeks. Irreversible cause of vitamin B12 deficiency

In general, patients with an irreversible cause should be treated continuously, whereas those patients with a reversible cause should be treated till the deficiency is corrected.

\section{PREVENTION}

Due to prolonged medication use screening of patient should be done

$>$ The average intake of vitamin $\mathrm{B}_{12}$ in the United States is $3.4 \mathrm{mcg}$ per day, and the recommended dietary allowance is $2.4 \mathrm{mcg}$ per day for adult, and 2.6 microgram per day for pregnant women

Fortified cereals prevent the vitamin B12 Deficiency

It is recommending that patients who have gone bariatric surgery should take $1 \mathrm{mg}$ of oral vitamin $\mathrm{B}_{12}$ per day indefinitely.

\section{CONCLUSION}

Deficiency of Vitamin B12 is a very old disease and research and current information also receive about $\mathrm{B}_{12}$ deficiency, the broad array of its effects, and methods for its diagnosis.

Due to vitamin B12 Deficiency production of all types of blood cells are affected but its effects extend to other tissues and organs, especially in the nervous system. Clinical picture suggest that diagnosis depends first on a high index of suspicion and then on the judicious application of appropriate testing.

\section{REFERENCES:}

01. The Many Faces of Cobalamin (Vitamin B12) Deficiency (nih.gov)

02. Vitamin B12 Deficiency - American Family Physician (aafp.org)

03. Vitamin B12 deficiency from the perspective of a practicing hematologist Blood American Society of Hematology (ashpublications.org)

04. Vitamin B12 or folate deficiency anaemia NHS (www.nhs.uk) 